\title{
Major approaches in improving wheat resistance to the crucially dangerous diseases in Kazakhstan
}

\author{
Rsaliyev A.S. ${ }^{1 *}$, Turuspekov Y. ${ }^{2}$, Abugalieva S. ${ }^{2}$, Amirkhanova N. ${ }^{1}$, Pahratdinova Z. ${ }^{1}$, \\ Rsaliyev Sh.S. ${ }^{3}$, Chudinov V. ${ }^{4}$, Gultyaeva E. ${ }^{5}$, Abugalieva A. ${ }^{3}$, Kokhmetova A. ${ }^{2}$, \\ Strochkov V. ${ }^{1}$, Yskakova G. ${ }^{1}$ \\ ${ }^{1}$ Research Institute for Biological Safety Problems, Zhambyl region, Kazakhstan \\ ${ }^{2}$ Institute of Plant Biology and Biotechnology, Almaty, Kazakhstan \\ ${ }^{3}$ Kazakh Research Institute of Farming and Crop Science, Almaty region, Kazakhstan \\ ${ }^{4}$ Karabalyk Agricultural Experimental Station, Kostanai region, Kazakhstan \\ ${ }^{5}$ All-Russian Institute of Plant Protection, St. Petersburg, Russia \\ *e-mail: aralbek@mail.ru
}

In Kazakhstan, rusts (Puccinia graminis, P. triticiana and P. striiformis) and leaf spots (Zymoseptoria tritici, Pyrenophora tritici-repentis, Parastagonospora nodorum) are most common among the crucially dangerous diseases of wheat. Since 2018 science and technology program "Development of the innovative systems for increasing the resistance of wheat varieties to especially dangerous diseases in the Republic of Kazakhstan" is realized in the country under financial support of the Ministry of Agriculture (program number BR06249329). The specialists in plant pathology, mycology, breeding, genetics and molecular biology from various scientific institutions take part in implementation of the program. As a result in 2018 sources of wheat resistance to the above mentioned diseases have been ascertained. Among the tested spring wheat cultivars 44 genotypes appeared to be resistant to rust and leaf spot species. New breeding lines (91) of spring wheat were created with use of disease resistance donors. Fifty two perspective cultivars of spring soft wheat from the breeding institutions of Kazakhstan and Russia were studied and the cultivars with effective combinations of Lr- and Sr-genes were revealed among them. The data of genotyping the collection of common wheat using new genomic technologies (Illumina 20K SNP array technology) were obtained. On the basis of a genome-wide association analysis of the soft wheat resistance to the most dangerous diseases at the adult plant stage, 11 QTLs associated with resistance to 4 fungal pathogens were identified. Alignment and analysis of nucleotide sequences of $Z$. tritici, $P$. tritici-repentis and $P$. nodorum genes resulted in identification of polymorphic sites that were used to develop species-specific primers ensuring highly sensitive diagnosing of all studied fungi by PCR. 